\title{
COMMUTANTS AND CYCLIC VECTORS
}

\author{
JAMES A. DEDDENS, ${ }^{1}$ RALPH GELLAR ${ }^{2}$ AND DOMINGO A. HERRERO ${ }^{3}$
}

\begin{abstract}
This note analyzes the relationship between various statements concerning the commutant of a bounded linear operator on a Hilbert space and the existence of cyclic vectors for the operator and its adjoint.
\end{abstract}

For a bounded linear operator $T$ on a Hilbert space $H, \mathscr{A}_{T}$ will denote the weakly closed algebra generated by $T$ and the identity $I$, and $\mathscr{A}_{T}^{\prime}$ will denote the commutant of $T$. We say that $T$ has a cyclic vector $x_{0}$ if $\left\{\mathscr{A}_{T} x_{0}\right\}$ is dense in $H$. For a finite dimensional operator or a normal operator the following are known to be equivalent (see [1]):

$\left(\mathrm{M}_{1}\right) T$ and $T^{*}$ have cyclic vectors.

$\left(\mathrm{M}_{2}\right) T$ or $T^{*}$ has a cyclic vector.

$\left(\mathrm{M}_{3}\right) \mathscr{A}_{T}^{\prime}$ is abelian.

In this note we analyze the relationships (this was asked by Professor B. Sz.-Nagy at the International Conference on Operator Theory, Indiana University, 1970) between the above statements and the following statements (which are also equivalent for finite dimensional operators):

$\left(\mathrm{M}_{4}\right) \mathscr{A}_{T}=\mathscr{A}_{T}^{\prime}$.

$\left(\mathrm{M}_{5}\right)$ Every invariant subspace for $T$ is hyperinvariant (i.e., invariant for $\left.\mathscr{A}_{T}^{\prime}\right)$.

Example 1. Let $T$ be the bilateral shift on $l^{2}$ defined by $T e_{n}=e_{n+1}$. Then $T$ satisfies $\mathbf{M}_{1}, \mathbf{M}_{2}, \mathbf{M}_{3}$ but not $\mathbf{M}_{4}, \mathbf{M}_{5}$ (see [3, Problems 115, 116], [4]).

Example 2 (Due to D. Sarason). Let $S$ be the unilateral shift on $l_{+}^{2}$ defined by $S e_{n}=e_{n+1}(n \geqq 0)$, and $T=S^{*} \oplus S^{*}$. Then $T$ satisfies $\mathrm{M}_{2}$ but not $M_{1}, M_{3}, M_{4}, M_{5}$ (see [3, Problem 126], [4]).

ExAmple 3. Let $B$ be the bilateral weighted shift on $l^{2}$ defined by $B e_{n}=2^{n} e_{n+1}(n<0)$ and $B e_{n}=e_{n+1}(n \geqq 0)$. Then $B$ is not invertible and hence $\mathscr{A}_{B}=\mathscr{A}_{B}^{\prime}$ [2], [7]. Moreover, $\Pi_{0}\left(B^{*}\right)=\{z: 0<|z|<1\}$. Therefore $B^{*}$ has a cyclic vector, but $B$ does not [6]. Let $T=(B+2) \oplus\left(B^{*}-2\right)$. The

Received by the editors April 23, 1972.

AMS (MOS) subject classifications (1970). Primary 47A05, 47B99.

Key words and phrases. Commutant, cyclic vectors, weighted shift.

${ }_{1}^{1}$ This research was partially supported by NSF Grant GP-16292.

${ }^{2}$ This research was supported by the North Carolina Engineering Foundation.

${ }^{3}$ This research was partially supported by NSF Grant GU-3171.

(C) American Mathematical Society 1974 
fact that the spectrum of $T$ is the disjoint union of the two discs comprising the spectrum of $B+2$ and $B^{*}-2$ implies that $[5, \S 5]$.

$$
\mathscr{A}_{T}^{\prime}=\mathscr{A}_{B+2}^{\prime} \oplus \mathscr{A}_{B^{*}-2}^{\prime}=\mathscr{A}_{B+2} \oplus \mathscr{A}_{B^{*}-2}=\mathscr{A}_{T} \text {. }
$$

If $(x, y)$ were a cyclic vector for $T$ (resp. $T^{*}$ ), then $x$ (resp. $y$ ) would be cyclic for $B . T$ satisfies $\mathrm{M}_{3}, \mathrm{M}_{4}, \mathrm{M}_{5}$ but not $\mathrm{M}_{1}, \mathrm{M}_{2}$.

The implications $M_{1} \Rightarrow M_{2}, M_{4} \Rightarrow M_{3}$, and $M_{4} \Rightarrow M_{5}$ are trivially true, and the Examples 1, 2, 3 show that any other implication is false, except perhaps

(1) Does $M_{1}$ imply $M_{3}$ ?

(2) (Due to Douglas and Pearcy [1]) Does $M_{5}$ imply $M_{3}$ or $M_{4}$ ? which are still open problems.

We close by remarking that Example 3 answers in the negative the question raised by the first author at the Conference on Operator Theory, Durham, 1971. That question was: Does $\mathscr{A}_{T}^{\prime}$ always have a cyclic vector? Our answer: Not necessarily, even when $\mathscr{A}_{T}^{\prime}=\mathscr{A}_{T}$.

\section{REFERENCES}

1. R. G. Douglas and C. Pearcy, On a topology for invariant subspaces, J. Functional Analysis 2 (1968), 323-341. MR 38 \#1547.

2. R. Gellar, Two sublattices of weighted shift invariant subspaces, Indiana Univ. Math. J. 23 (1973), 1-10.

3. P. R. Halmos, A Hilbert space problem book, Van Nostrand, Princeton, N.J., 1967. MR $34 \# 8178$.

4. H. Helson, Lectures on invariant subspaces, Academic Press, New York, 1964. MR 30 \#1409.

5. D. A. Herrero and N. Salinas, nalytically invariant and biinvariant subspaces, Trans. Amer. Math. Soc. 173 (1972), 117-136.

6. D. A. Herrero, Eigenvectors and cyclic vectors for bilateral weighted shifts, Rev. Un. Mat. Argentina. 26 (1972), 24-41.

7. T. R. Turner, Double commutants of singly generated operator algebras, Dissertation, University of Michigan, Ann Arbor, Mich., 1971.

Department of Mathematics, University of Kansas, Lawrence, Kansas 66044

Department of Mathematics, North Carolina State University, Raleigh, North Carolina 27607

Current address (Domingo A. Herrero): Universidad Nacional de Rio IV, Provincia de Córdoba, Republica Argentina 